\title{
Progresso da cercosporiose da beterraba sob diferentes regimes de pulverização
}

\author{
Progress of beet Cercospora leaf spot under different spraying regimes
}

\author{
Leandro Luiz Marcuzzo*, Sheila Chaiana Harbs, Bruna Kotkoski, Aline Cristina Paulakoski \\ Instituto Federal Catarinense, Rio do Sul, SC, Brasil. Autor para correspondência: leandro.marcuzzo@ifc.edu.br
}

Submissão: 06/02/2019 / Aceite: 06/03/2020

\begin{abstract}
RESUMO
A cercosporiose da beterraba, causada por Cercospora beticola Sacc. é controlada por meio de pulverizações foliares com fungicidas seguindo um calendário fixo, sem considerar o progresso da doença. Um sistema de previsão pode predizer o progresso da doença e direcionar o regime de pulverizações, reduzindo o número de aplicações e otimizando o manejo da doença. Com o objetivo de avaliar o progresso da cercosporiose sob os diferentes regimes de pulverização foi aplicada a técnica de modelagem estatística conhecida por modelos mistos. Estes modelos não incluem apenas os efeitos fixos, mas também os efeitos aleatórios para cada um dos indivíduos da população em estudo. Nas safras agrícolas de 2018 e 2019, os regimes de pulverização utilizados foram baseados em sistemas com valores de severidade estimada (SE) acumulada de 0,$15 ; 0,25$, e 0,35 , além dos controles padrões com intervalos de pulverizações de cinco e sete dias. A severidade acumulada da cercosporiose em função do tempo, nos cinco regimes de pulverização, foi calibrada com um modelo Gompertz ajustado pelo modelo misto e o efeito aleatório ajustado à assíntota superior. Como resultado da calibração do modelo, o tratamento com regime de pulverização com $\mathrm{SE}=0,35$ não diferiu dos controles padrões com relação à área abaixo da curva de progresso da doença (AACPD), severidade final e produtividade. Os dados apresentados nesse trabalho demonstram a eficiência do sistema de previsão no manejo da cercosporiose em beterraba, com a vantagem de reduzir o número de pulverizações com fungicidas e o impacto sobre o ambiente.
\end{abstract}

PALAVRAS-CHAVE: Beta vulgaris ssp. vulgaris L., Cercopora beticola, epidemiologia, controle químico.

\begin{abstract}
Cercospora leaf spot (CLS) caused by Cercospora beticola Sacc. is controlled by foliar spraying with fungicides following a fixed schedule, without considering the disease progress. A forecasting system can predict the progress of the disease and direct the spraying regime, reducing the number of applications and optimizing the disease management. To evaluate the progress of CLS under the different spraying regimes, the statistical modeling technique known as mixed models was applied. These models include not only fixed effects but also random effects for each individual in the studied population. In the harvest seasons of 2018 and 2019, the spraying regimes used were based on systems with accumulated estimated severity (SE) values of $0.15 ; 0.25$, and 0.35 , in addition to standard controls with spraying intervals of five and seven days. The accumulated severity of CLS as a function of time in the five spraying regimes was calibrated with a Gompertz model adjusted by the mixed model and the random effect adjusted to the upper asymptote. As a result of the model calibration, the spraying regime treatment with $\mathrm{SE}=0.35$ did not differ from the standard controls regarding the area under the disease progress curve (AACPD), final severity, and productivity. The data presented in this study demonstrate the efficiency of a forecasting system in CLS management, with the advantage of reducing the number of fungicide sprayings and environmental impact.
\end{abstract}

KEYWORDS: Beta vulgaris ssp. vulgaris L., Cercopora beticola, epidemiology, chemical control.

\section{INTRODUÇÃO}

A cultura da beterraba vermelha ou de mesa (Beta vulgaris ssp. vulgaris L.) ocupa destaque entre as hortaliças de maior expressão econômica do Brasil. Essa cultura constitui atividade socioeconômica de grande relevância para pequenos produtores da região sul, e em especial na região do Alto Vale do Itajaí 
em Santa Catarina, com uma área de aproximadamente 1600 hectares (EPAGRI 2015).

Diversos são os fatores que contribuem para a baixa produtividade da cultura, com destaque para as doenças de diversas etiologias, que podem causar danos significativos à cultura. Dentre as doenças, a cercosporiose, causada por Cercospora beticola Sacc. é uma doença de grande importância na região do Alto Vale do Itajaí. Essa região apresenta condições favoráveis de temperatura $\left(\geq 22{ }^{\circ} \mathrm{C}\right)$ e umidade relativa do $\operatorname{ar}(\geq 90 \%)$ para a ocorrência da doença. A cercosporiose pode incidir em toda a parte aérea da planta, que consequentemente acaba comprometendo a produtividade (FERREIRA \& TIVELLI 1989, TIVELLI et al. 2011).

Vale destacar também que os tratamentos fitossanitários utilizados na cultura da beterraba são realizados de forma inadequada, sem saber se há necessidade ou não de aplicar fungicidas, utilizando normalmente aplicações semanais com base em calendários fixos; essa prática resulta em número excessivo de aplicações e consequente contaminação do ambiente (DELLAMATRICE 2000). Segundo BERGAMIN FILHO \& AMORIM (2011) os sistemas de previsão de doenças de plantas são representações simplificadas da realidade e preveem o início ou o desenvolvimento futuro de uma doença (KRAUSE \& MASSIE 1975, BARRETO et al. 2004, REIS 2004), além da previsão do momento adequado à pulverização com fungicidas, considerando que o patógeno se encontra em quantidades suficientes para iniciar uma epidemia e que o hospedeiro seja suscetível (MIZUBUTI 1999).

$\mathrm{Na}$ Europa, existe o sistema de previsão da cercosporiose da beterraba açucareira denominado Cercobet, que passou por modificações e encontra-se na terceira versão (RACCA \& JÖRG 2007). O sistema envolve vários fatores ambientais e da cultura e considera apenas a temperatura e a pressão de vapor como modelo, mas não diretamente o principal fator que é o binômio temperatura versus molhamento foliar. Sistemas simplificados que analisam a temperatura e a duração do molhamento foliar são de fácil funcionalidade e de interpretação dos dados pelos agricultores.

No estudo de epidemias, a dinâmica temporal das doenças de plantas é enfatizada, pois o progresso de doenças é frequentemente a manifestação mais facilmente visualizada (CAMPBELL \& MADDEN 1990). $\mathrm{Na}$ análise temporal de doenças, a análise da curva de progresso é a melhor representação de uma epidemia e a interpretação do formato dessas curvas e sua intensidade final são fundamentais para se efetuar o manejo de doenças (BERGAMIN FILHO \& AMORIM 1996).

Modelos não lineares, comumente usados para representar crescimento de epidemias, como o Logístico e o de Gompertz podem se comportar de forma diferente conforme o número de repetições, o tratamento e unidade experimental. Mediante isso, os dados observados numa mesma unidade experimental ao longo do tempo são modelados mais adequadamente por modelos de efeitos mistos, pois nesses casos, a análise de variância clássica leva à violação da independência entre as observações. Por outro lado, os modelos não lineares de efeitos mistos permitem explorar e modelar adequadamente a variabilidade de dados com erros aleatórios coletados ao longo do tempo e, possivelmente com algum nível de correlação entre si no progresso de uma epidemia (DAVIDIAN \& GILTINAN 2003).

O objetivo deste trabalho foi analisar o progresso da cercosporiose da beterraba utilizando modelos mistos e identificar qual o regime de pulverização baseado em um sistema de previsão é o mais eficiente comparado ao sistema convencional de controle.

\section{MATERIAL E MÉTODOS}

\section{Detalhamento do Experimento}

O trabalho foi conduzido no Instituto Federal Catarinense - IFC/Campus Rio do Sul, no município de Rio do Sul, SC, (2711'07' S e 49³9'39" W, altitude 655 metros) durante o período de 14 de setembro a 15 de dezembro de 2018 e de 16 de setembro a 14 de dezembro de 2019 totalizando 13 semanas após a semeadura.

Os dados meteorológicos foram obtidos de uma estação Davis ${ }^{\circledR}$ Vantage Vue $300 \mathrm{~m}$ localizada ao lado do experimento e os dados médios durante a condução do experimento foram de 18,9 e 19,2 ${ }^{\circ} \mathrm{C}$ para temperatura do ar; de 14,5 e 14 horas de umidade relativa do $a r \geq 90 \%$ e a precipitação pluvial acumulada foi de 300 e 446,5 mm respectivamente para 2018 e 2019.

Segundo a classificação de Köeppen, o clima local é subtropical úmido (Cfa) e solo classificado como Cambissolo Háplico Tb distrófico (EMBRAPA 2013) com os seguintes atributos químicos: pH em água de 6,0; teores de $\mathrm{Ca}^{+2}, \mathrm{Mg}^{+2}, \mathrm{Al}^{+3}$ e CTC de 4,2; 1,8; 0,0 e 9,54 $\mathrm{cmolc} \mathrm{dm}^{-3}$, respectivamente; saturação por bases de $66,49 \%$, teor de argila de $30 \% \mathrm{~m} / \mathrm{v}$ e teores de $\mathrm{P}$ e K de $14 \mathrm{e} 134 \mathrm{mg} \mathrm{dm}^{-3}$, respectivamente.

Sementes de beterraba do cultivar Boro foram semeadas a campo em quatro repetições constituídas de uma área de 1,5 x 1,25 m utilizando cinco linhas com espaçamento de $25 \mathrm{~cm}$ entre linhas e $10 \mathrm{~cm}$ entre 
plantas com 40 plantas $\mathrm{m}^{-2}$ (equivalente a 400.000 plantas ha $\mathrm{h}^{-1}$ ), ficando com um estande final de 75 plantas em cada repetição. A calagem, adubação, tratos culturais seguiram as normas da cultura (TIVELLI et al. 2011).

Para que houvesse inóculo na área, mudas de beterraba cv. Boro com 30 dias de idade foram inoculadas com auxílio de um atomizador portátil uma suspensão de $10^{4}$ conídios de $C$. beticola. Após 24 horas de câmara úmida as mudas foram transplantadas a cada um metro linear ao redor do experimento no dia da semeadura.

Para o controle da cercosporiose foi feita a aplicação da mistura de fungicidas protetores mancozeb $(80 \%)+$ oxicloreto de cobre $(50 \%)$ na dose de $250 \mathrm{~g}+200 \mathrm{~g} \mathrm{pc}^{-1} \mathrm{~h}^{-1}$ baseado no modelo descrito por MARCUZZO et al. (2016b) expresso em SE $=0,0001105 *\left(\left((x-8)^{2,294387}\right) *\left((36-x)^{0,955017}\right)\right)$ * $\left(0,39219 /\left(1+25,93072{ }^{*} \exp \left(-0,16704^{*} y\right)\right)\right)$, onde SE, representa o valor da severidade estimada $(0,1$ ou seja, percentual da doença $/ 100) ; x$ a temperatura $\left({ }^{\circ} \mathrm{C}\right)$ e $y$ o molhamento foliar (horas) que foi avaliado através da umidade relativa do ar $\geq 90 \%$.

Os tratamentos foram constituídos de valores de SE de 0,$15 ; 0,25$ e 0,35 e comparados com sistema convencional com pulverização a cada cinco e sete dias. A pulverização no sistema de previsão foi realizada quando o somatório diário dos valores de $\operatorname{SE}(15,25,35)$ foi atingido, sendo então zerado o somatório e iniciada nova soma dos SE. A cada ocorrência de $25 \mathrm{~mm}$ de chuva, todos os tratamentos eram pulverizados, zerados e reiniciava-se a contagem do somatório dos valores de severidade.

As pulverizações iniciaram-se a partir dos 21 dias após a semeadura e foram efetuadas com um pulverizador costal eletrônico Jetbras ${ }^{\circledR}$ calibrado para $400 \mathrm{~L} \mathrm{ha}^{-1}$.

\section{Avaliação da cercosporiose}

A severidade da doença foi avaliada através de escala diagramática proposta por MAY DE MIO et al. (2008) em cada folha presente na planta a intervalos regulares de sete dias em dez plantas previamente demarcadas por repetição. A severidade da doença ao longo do ciclo foi integralizada e calculada a área abaixo da curva de progresso da doença (AACPD), através da fórmula: AACPD $=\Sigma[(y 1+y 2) / 2]^{*}(t 2-t 1)$, onde $y 1$ e $y 2$ refere-se a duas avaliações sucessivas da severidade da doença realizadas no tempo $t 1$ e $t 2$ ( 7 dias), respectivamente.

\section{Análise dos Dados}

Modelos não lineares utilizando o logistico e Gompertz foram usados para descrever o progresso da cercosporiose no tempo em cada um dos tratamentos.

Após examinar as curvas de tendência em cada tratamento foi escolhido o modelo de Gompertz como adequado para representar a severidade acumulada da cercosporioseem semanas após a semeadura. Os valores observados foram ajustados de acordo com as curvas de tendência do modelo de Gompertz definido por:

$$
y_{i j}=a \times \exp \left(-b \times c x_{i}\right)
$$

onde, $y_{i j}$ representa a severidade (\%) na semana após o transplantio para o tratamento j, $x_{i}$ é o número de semanas após o transplantio, $a$, o parâmetro a representa a assíntota superior, $b$, o ponto de inflexão e $c$ é o parâmetro que indica a taxa de progresso. Os parâmetros estimados no modelo de efeitos fixos foram utilizados posteriormente para o ajuste do modelo misto não linear (DAVIDIAN \& GILTINAN 2003). Nesse caso, o modelo de Gompertz foi redefinido de acordo com a seguinte fórmula:

$$
y_{i j}=\left(a+u_{a j}\right) \times \exp \left(-b \times c x_{i}\right)+e_{i j}
$$

onde $u_{a j}$ representa o efeito aleatório adicionado à assíntota para a representação individual de cada tratamento enquanto $e_{i j}$ representa o erro experimental.

Nesta análise, a comparação de diferentes modelos com as combinações possíveis entre os efeitos fixos $(a, b$ e $c)$ e os aleatórios $\left(u_{\mathrm{aj}}, u_{b j}\right.$ e $\left.u_{c j}\right)$ permitiu selecionar a adição de um único efeito aleatório para a assíntota do modelo não linear misto. O melhor ajuste foi obtido com o modelo representado pela equação 2. Os critérios utilizados para a seleção deste modelo foram o logaritmo da máxima verossimilhança (logLik), o critério de informação de Akaike (AIC), o critério de informação Bayesiano (BIC) e do desvio. Estes critérios são comumente usados para comparar o grau de ajuste entre os modelos, com o melhor modelo que apresentam os menores valores em todos esses critérios (SAKAMOTO et al. 1986).

Uma vez que os valores iniciais foram encontrados, o modelo misto não-linear foi ajustado com o nlmer que é um procedimento incluído no pacote Ime4 (BATES et al. 2012) usando o método conhecido 
como aproximação de Laplace para o ajuste do modelo. Para a visualização, foi usado o pacote lattice (DEEPAYAN 2012). Ambos os pacotes fazem parte do software $R$ de análise estatística versão 2.15 .1 ( $R$ DEVELOPMENT CORE TEAM 2012).

As colheitas das plantas demarcadas foram realizadas aos 91 e 88 dias após a semeadura, respectivamente, em cada ano, quando foram pesadas e posteriormente convertidas para produtividade comercial em quilogramas por hectare $\left(\mathrm{Kg} \mathrm{ha}^{-1}\right)$.

As médias da AACPD e produtividade entre os regimes de pulverização foram submetidas à análise de variância pelo teste de $\mathrm{F}$ e quando significativas foram comparadas pelo teste de Tukey $5 \%$.

\section{RESULTADOS E DISCUSSÃO}

Nas safras de 2018 e 2019, os sistemas de previsão $S E=0,15$; $S E=0,25 ; S E=0,35$; e os regimes de pulverização a cada cinco e sete dias resultaram em 10 e 9; 6 e 4; 5 e 3; 10 e 10; e 7 e 7 pulverizações, respectivamente (Tabela 1). Pode-se constatar que o sistema $\mathrm{SE}=0,35$ apresentou o menor número de pulverizações em ambos os anos de avaliação e o sistema $\mathrm{SE}=0,15$ apresentou o mesmo número de pulverizações quando realizado a cada cinco dias em 2018 e de apenas uma pulverização a menos em 2019 (Tabela 1).

Tabela 1. Número de pulverizações em diferentes programas de manejo da cercosporiose em beterraba. IFC/Campus Rio do Sul, 2018 e 2019.

Table 1. Number of sprayings in different beet Cercospora leaf spot management programs. IFC/Rio do Sul Campus, 2018 and 2019.

\begin{tabular}{lrc}
\hline \multirow{2}{*}{ Tratamento } & \multicolumn{3}{c}{ Número de pulverizações } \\
\cline { 2 - 3 } SE $=0,15$ & 2018 & 2019 \\
SE $=0,25$ & 10 & 9 \\
SE $=0,35$ & 6 & 4 \\
Cinco dias & 5 & 3 \\
Sete dias & 10 & 10 \\
\end{tabular}

SE: representa o valor da severidade estimada (0,1 ou seja, percentual da doença /100); Cinco e sete dias corresponde ao intervalo de tempo entre as pulverizações.

Quando comparado ao sistema de aplicação a cada sete dias, o sistema $\mathrm{SE}=0,35$ resultou em redução no número de pulverizações de $28 \%$ e $57 \%$ nos anos de 2018 e 2019 , respectivamente (Tabela 1).

$\mathrm{Na}$ Europa existe um sistema de previsão da cercosporiose da beterraba açucareira denominado Cercobet. Esse sistema de previsão passou por modificações e encontra-se na terceira versão (RACCA \& JÖRG 2007). No entanto, a literatura apenas expressa o quanto o sistema coincidiu com a ocorrência da doença, mas não descreve se houve a redução do número de pulverizações.

$\mathrm{Na}$ área abaixo da curva de progresso da doença (AACPD), os sistemas não foram significativos em ambos os anos de avaliação em relação aos sistemas de pulverização a cada cinco e sete dias, evidenciando que é possível realizar a redução do número de pulverizações em relação ao sistema convencional de controle (Tabela 2). As pulverizações com fungicidas, baseadas nos sistemas de previsão $\mathrm{SE}=0,35$ e $\mathrm{SE}=0,25$, resultaram no manejo efetivo da doença. Os valores de AACPD obtidos nesse trabalho ficaram acima dos encontrados por MARCUZZO et al. (2016a) na cultura da beterraba quando avaliaram o uso da mistura de mancozeb+oxicloreto de cobre em controle convencional da doença na mesma região de estudo. Essa diferença deve-se provavelmente a época de cultivo e a utilização plantas infectadas que serviram como inóculo no presente trabalho.

De maneira geral, o progresso da doença entre os regimes de pulverização ocorreu em níveis de severidade próximos após o início da doença e durante o desenvolvimento da cultura (Figura 1). No entanto, as epidemias não diferiram (Figura 2A) entre os anos, apesar da pouca sobreposição da assíntota, pois os sintomas e avaliação da severidade iniciaram a partir da $4^{a}$ semana após a semeadura nos dois anos de avaliação e a severidade seguiu de forma acentuada e contínua ao longo do ciclo da cultura em relação aos diferentes regimes de pulverização (Figura 1).

Os tratamentos não diferiram na severidade final (Tabela 2) ficando entre 11,47 a 12,75\% em $2018 \mathrm{e}$ de 9,98 a 10,25\% em 2019. Valores esses acima dos encontrados por MARCUZZO et al. (2016a), quando avaliaram a severidade final em pulverização a cada sete dias, utilizando a mistura de mancozeb+oxicloreto de cobre. A severidade final da doença pode variar conforme o local, época de cultivo e da pressão de 
inóculo na área experimental.

Tabela 2. Área abaixo da curva de progresso da doença (AACPD), severidade final (\%) da cercosporiose e produtividade $\left(\mathrm{Kg} \mathrm{ha}^{-1}\right)$ da cultura da beterraba em diferentes regimes de pulverização. IFC/Campus Rio do Sul, 2018 e 2019.

Table 2. Area under disease progress curve (AUDPC), final severity (\%) of Cercospora leaf spot, and productivity $\left(\mathrm{Kg} \mathrm{ha}^{-1}\right)$ of beet crops in different spraying regimes. IFC/Rio do Sul Campus, 2018 and 2019.

\begin{tabular}{lllllll}
\hline Tratamento & \multicolumn{2}{c}{ AACPD } & \multicolumn{2}{c}{ Severidade final (\%) } & \multicolumn{2}{c}{ Produtividade $\left(\mathrm{Kg} \mathrm{ha}^{-1}\right)$} \\
\cline { 2 - 7 } & 2018 & 2019 & 2018 & \multicolumn{1}{c}{2019} & \multicolumn{1}{c}{2018} & 2019 \\
\hline SE=0,15 & $95,50 \mathrm{~ns}$ & $282,91 \mathrm{~ns}$ & $11,76 \mathrm{~ns}$ & $9,98 \mathrm{~ns}$ & $35.941 \mathrm{~ns}$ & $40.753 \mathrm{~ns}$ \\
SE=0,25 & 92,91 & 289,25 & 11,51 & 10,16 & 48.025 & 37.535 \\
SE=0,35 & 109,01 & 289,65 & 12,75 & 10,25 & 43.779 & 45.283 \\
Cinco dias & 125,87 & 285,45 & 11,99 & 10,09 & 39.057 & 26.544 \\
Sete dias & 101,92 & 285,35 & 11,47 & 10,17 & 44.322 & 40.715 \\
\hline CV (\%) & 16,02 & 3,61 & 6,20 & 3,24 & 32,80 & 31,3 \\
\hline
\end{tabular}

ns = não significativo pelo teste $\mathrm{F}$.

Como não houve diferença de dias para o início da epidemia entre os tratamentos (Figura 1) em ambos os anos, as repetições tiveram o mesmo padrão de avaliação nos diferentes anos e não diferiram, confirmado pela sobreposição longitudinal do desvio da assíntota (Figura 2A e Figura 2B). Ao se comparar o intervalo das médias nas diferenças da assíntota (Figura $2 \mathrm{C}$ ) sobre o efeito dos regimes de pulverização, verificou-se que todos se sobrepõem, não havendo diferenças entre eles. Como $S E=0,35$ sobrepõe no desvio padrão de $S E=0,25$ e $S E=0,15$, portanto não diferem estatisticamente. Verifica-se também que a pulverização a cada cinco e sete dias estão sobrepostas entre si, evidenciando que não há diferença entre esses dois programas convencionais de pulverização.
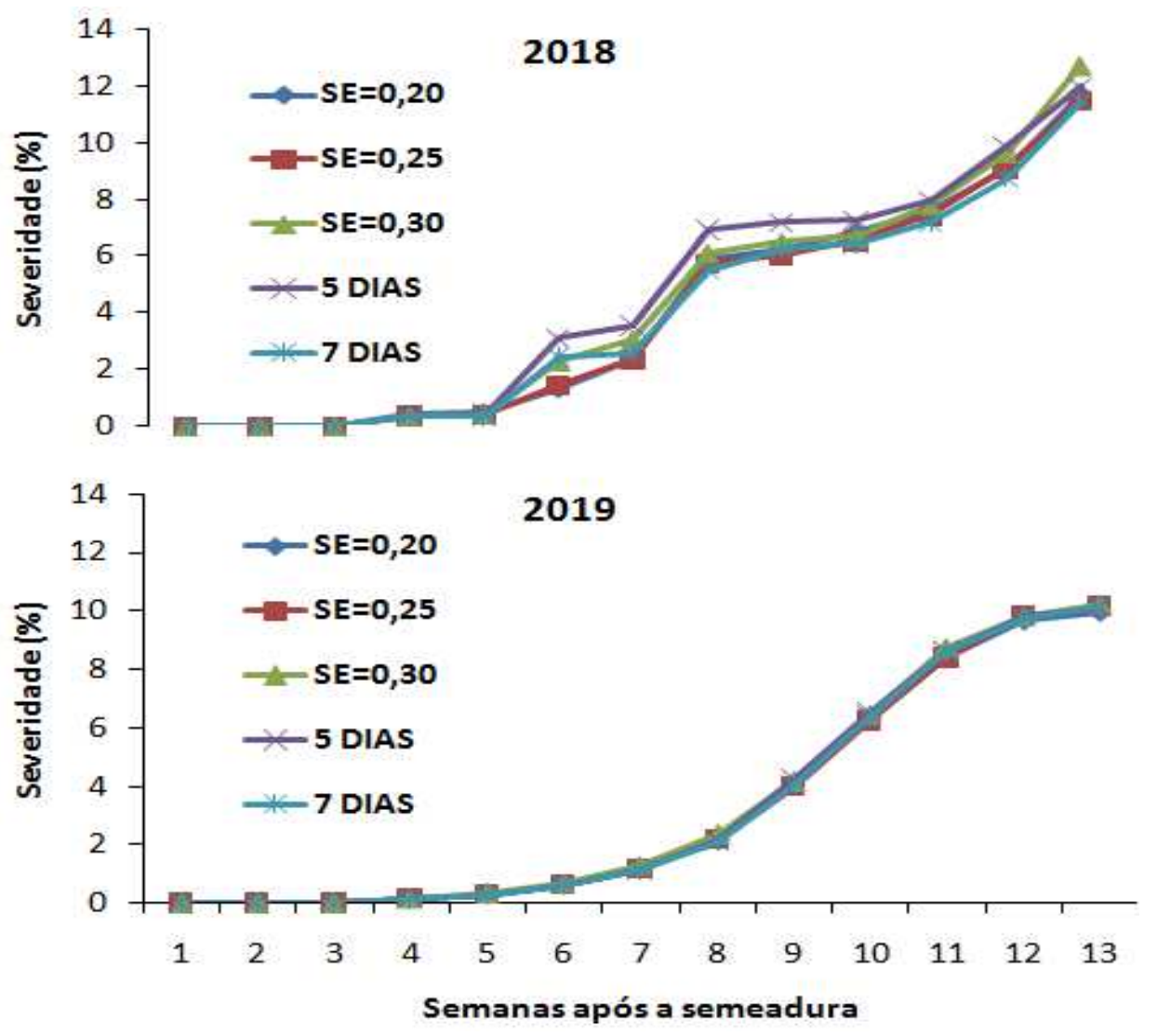

Figura 1. Progresso da severidade da cercosporiose da beterraba sob diferentes programas de pulverização. IFC/Campus Rio do Sul, 2018 e 2019.

Figure 1. Severity progress of beet Cercospora leaf spot under different spraying programs. IFC/Rio do Sul Campus, 2018 and 2019. 

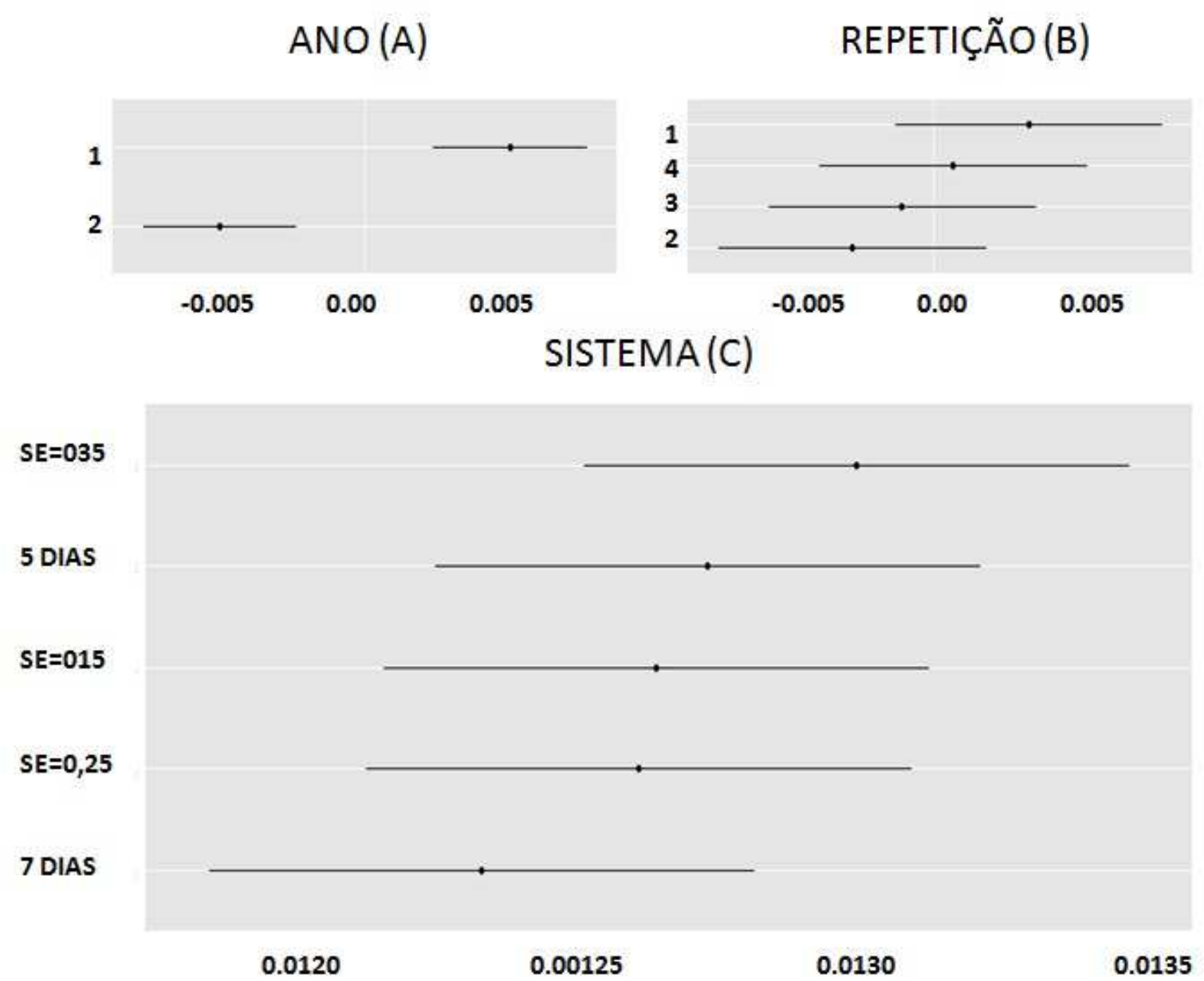

Figura 2. Diferenças na assíntota entre anos $(A)$, repetições $(B)$ e dados de severidade $(C)$ da cercosporiose da beterraba sob diferentes regimes de pulverização. IFC/Campus Rio do Sul, 2018 e 2019.

Figure 2. Differences in asymptote between years $(A)$, replicates $(B)$, and severity data $(C)$ of beet Cercospora leaf spot under different spraying regimes. IFC/Rio do Sul Campus, 2018 and 2019.

No presente trabalho, constatou-se que no sistema de previsão utilizado como indicador do momento da pulverização, o tratamento $\mathrm{SE}=0,35$ resultou em redução no número de pulverizações sem comprometer a produtividade (Tabela 2), pois em 2018 e 2019 a produtividade nesse sistema não diferiu estatisticamente dos controles convencionais a cada cinco e sete dias.

O uso do modelo utilizado para previsão tem por objetivo verificar se o comportamento do sistema real é coerente com o modelo avaliado (KRAUSE \& MASSIE 1975, KRANZ \& HAU 1980, BERGAMIN FILHO \& AMORIM 1996). Segundo TENG (1985), o rigor da avaliação de um sistema de previsão não deve ser exagerado, uma vez que o sistema é uma aproximação da realidade e durante o processo de avaliação, se necessário, pode se ter um espaço entre o que é estimado e o real para o aprimoramento contínuo do sistema (FERNANDES \& MAFFIA 1994).

\section{CONCLUSÃO}

O progresso temporal da cercosporiose da beterraba em função do regime de pulverização baseado em um modelo de previsão com $\mathrm{SE}=0,35$ não diferiu dos controles padrões e na produtividade do sistema convencional utilizado pelo produtor, com a vantagem de reduzir o número de pulverizações e com a mesma eficiência de controle, servindo como subsidio na tomada de decisão no manejo integrado da doença na cultura.

\section{AGRADECIMENTOS}

O apoio financeiro recebido do Conselho Nacional de Desenvolvimento Científico e Tecnológico $\mathrm{CNPq}$ - Brasil pela bolsa de iniciação científica Pibic e ao técnico de campo, Marcio Rampelotti pela implantação do experimento e execução das pulverizações. 


\section{REFERÊNCIAS}

BARRETO M et al. 2004. Sistemas de previsão e estação de aviso. In: VALE FXR et al. Epidemiologia aplicada ao manejo de doenças de plantas. Belo Horizonte: Perffil. p.243-266.

BATES D et al. 2012. Ime4: Linear mixed-effects models using S4 classes. R package. Vienna: R Foundation for Statistical Computing. Disponível em: http://www.R-project.org. Acesso em: 15 dez. 2018.

BERGAMIN FILHO A \& AMORIM L. 2011. Sistemas de previsão e avisos. In: AMORIM L et al. Manual de fitopatologia: princípios e conceitos. 4.ed. São Paulo: Ceres. p.389-408.

BERGAMIN FILHO A \& AMORIM L. 1996. Doenças de plantas tropicais: epidemiologia e controle econômico. São Paulo: Ceres. 289p.

CAMPBELL CL \& MADDEN LV. 1990. Introduction to plant disease epidemiology. New York: Wiley Interscience. 532p.

DAVIDIAN M \& GILTINAN DM. 2003. Nonlinear Models for Repeated Measurement Data: An Overview and Update. Journal of Agricultural, Biological, and Environmental Statistics 8: 387-419.

DEEPAYAN S. 2012. Lattice: Multivariate Data Visualization with R. Vienna: R Foundation for Statistical Computing. Disponível em: http://www.R-project.org. Acesso em: 15 dez. 2018.

DELLAMATRICE PM. 2000. Degradação do herbicida 14C Diuron por Acinetobacter baumannii e pela microbiota do solo. Dissertação (Mestrado em Ciências) Piracicaba: USP. 88p.

EMBRAPA. 2013. Empresa Brasileira de Pesquisa Agropecuária. Sistema Brasileiro de classificação de solos. 3.ed. Brasília: Embrapa. 353p.

EPAGRI. 2015. Levantamento de espécies de hortaliças cultivadas na região do Alto Vale do Itajaí, 2010 e 2014. Ituporanga: Epagri. 7p. (Relatório de pesquisa).

FERNANDES JMC \& MAFFIA LA. 1994. Simulação de epidemias. Revisão Anual de Patologia de Plantas 2: $293-334$.

FERREIRA MD \& TIVELLI SW. 1989. Cultura da beterraba: recomendações gerais. Guaxupé: Cooxupé. 14p. (Boletim Técnico 2).

KRANZ J \& HAU B. 1980. Systems analysis in epidemiology. Annual Review of Phytopathology 18: 67-83.

KRAUSE RA \& MASSIE LB. 1975. Predictive systems: modern approaches to disease control. Annual Review of Phytopathology 13: 31-47.

MAY DE MIO LL et al. 2008. Proposta de escala diagramática para quantificação da cercosporiose da beterraba. Scientia Agraria 9: 331-337.

MARCUZZO LL et al. 2016a. Efeito de fosfito de potássio e de fungicidas no controle da cercosporiose (Cercospora beticola) da beterraba. Summa Phytopathologica 42: 186-187.

MARCUZZO LL et al. 2016b. Influence of temperature and leaf wetness duration on the severity of Cercospora leaf spot of beet. Summa Phytopathologica 42: 89-91.

MIZUBUTI ESG. 1999. Sistema de previsão de doenças de plantas: uma ferramenta útil? In: ZAMBOLIM L. $1^{\circ}$ Encontro de manejo integrado de doença e pragas. Viçosa: UFV. p.42-46.

R DEVELOPMENT CORE TEAM. 2012. R: A language and environment for statistical computing. Vienna: R Foundation for Statistical Computing. Disponível em: http://www.R-project.org. Acesso em: 2 jul. 2018.

RACCA P \& JÖRG E. 2007. CERCBET 3 - a forecaster for epidemic development of Cercospora beticola. Bulletin OEPP/EPPO 37: 344-349

REIS EM. 2004. Previsão de doenças de plantas. Passo Fundo: UPF. 316p.

SAKAMOTO Y et al. 1986. Akaike Information Criterion Statistics. Dordrecht: Kluwer Academic Publishers. 290p.

TENG PS. 1985. Comparison of simulation approaches to epidemic modeling. Annual Review of Phytopathology 23: 351-379.

TIVELLI SW et al. 2011. Beterraba, do plantio à comercialização. Campinas: Instituto Agronômico. 45p. (Boletim Técnico 210). 